\title{
RESEARCH
}

\section{Impact of an electronic link between the emergency department and family physicians: a randomized controlled trial}

\author{
Eddy Lang, Marc Afilalo, Alain C. Vandal, Jean-François Boivin, Xiaoqing Xue, Antoinette Colacone, \\ Ruth Léger, Ian Shrier, Stephen Rosenthal
}

Fast-tracked article. Published at www.cmaj.ca on Jan. 6, 2006.

\section{ABSTRACT}

Background: Electronic information exchange is believed to improve efficiency and reduce resource utilization. We developed a Web-based standardized communication system (SCS) that enables family physicians to receive detailed reports of their patients' care in the emergency department. We sought to determine the impact of the SCS on measures of resource utilization in the emergency department and family physician offices.

Methods: We used an open 4-period crossover clusterrandomized controlled design. During 2 separate 10 -week intervention phases, family physicians received detailed reports of their patients' emergency department visits over the Internet, and in the alternating control phases they received a 1-page copy of the emergency department notes by mail. The primary outcome was the number of repeat visits to the emergency department within 14 days of the initial visit. Secondary outcomes included duplication of test and specialty consultation requests by the emergency and family physician. Outcomes were measured using the hospital database and questionnaires sent to the family physicians.

Results: A total of 2022 patient visits to the emergency department from 23 practices were used in the study. Use of the SCS failed to reduce the number of repeat visits to the emergency department within 14 days (odds ratio [OR] 1.10, $95 \%$ confidence interval $[\mathrm{Cl}]$ o.8-1.51) and 28 days (OR 1.01, $95 \% \mathrm{Cl} 0.8-1.27)$. There was no significant duplication of requests for diagnostic tests between the emergency and family physician during the intervention and control phases (24 v. $22, p=0.93$ ), but there was significantly greater duplication in specialty consultation requests in the intervention phase than in the control phase (20 v. 8, $p=0.049$ ).

Interpretation: An electronic link between emergency and family physicians did not result in a significant reduction in resource utilization at either service point. Investments in improved electronic information exchange between emergency departments and family physician offices may not be substantiated by a reduction in resource utilization.

CMAJ 2006;174(3):313-8
A lack of information exchange and communication between community-based health care providers and hospitals has been cited as an area of concern within the Canadian health care system..$^{1,2}$ Electronic health records have the potential to facilitate patient care and benefit clinicians by improving access to information at various service points within the system. Health care systems all over the world are investing heavily in improving information access and exchange among multiple providers. ${ }^{3}$

A breakdown in the exchange of clinical information between emergency departments and family physician offices can be deleterious for patient care. ${ }^{2}$ Family physicians are often required to manage therapy with missing or incomplete information for patients who have been discharged from hospital. ${ }^{4}$ Research in this domain ${ }^{5-7}$ has shown that the information elements most desired by family physicians are medications at discharge (new or changes to existing regimens), treatments given, laboratory test results, imaging and specialty consultation reports, and follow-up plans. The results of a recent survey in Ontario showed that $86 \%$ of emergency department chiefs thought that communication with community physicians needed improvement. $^{8}$

Electronic data exchange between hospitals, specialists and family physicians as it pertains to the care of patients with chronic medical conditions has been introduced. ${ }^{9}$ However, there have been no published reports of electronic linkage between emergency and family physicians. It seems plausible that enhanced information exchange would translate into less duplication and more efficiency in the use of precious and often costly medical resources.

We sought to determine the effect of enhanced electronic transfer of clinical information to family physicians regarding the care their patients received in the emergency department. Specifically, we set out to determine whether an electronic link would reduce resource utilization as measured by repeat visits to the emergency department and admissions to hospital while leading to more efficient use of diagnostic resources in the emergency department and family physician offices. 


\section{Methods}

The study design was an open (unblinded) 4-period crossover cluster-randomized controlled trial of 23 family physician practices.

The study was performed in the emergency department of the Sir Mortimer B. Davis-Jewish General Hospital (SMBDJGH) in Montreal, an adult university teaching hospital with 637 beds. The emergency department's annual census for 2000-200I was about 60000 visits.

Family physicians were selected for recruitment on the basis of the frequency with which their patients consulted the hospital's emergency department; a minimum of Ioo annual visits per physician clientele was required. Physicians were recruited by telephone, letters of invitation and information sessions. Consenting physicians, rather than patients or visits, were then randomly assigned to the initial intervention or control group to prevent contamination of effect between the intervention and control phases (i.e., so that family physicians would not receive information through electronic and conventional means on different patients at the same time). To ensure balance between the 2 study arms, family physician practices underwent stratified randomization on the basis of the mean age $(<65 \mathrm{v} . \geq 65$ years $)$ and annual rates of emergency department visits $(<200 \mathrm{v}$. $\geq 200)$ of their clientele. Stratified randomization was achieved by a separate randomization procedure performed within each of the strata. ${ }^{10} \mathrm{~A}$ computer-generated random allocation sequence was concealed until all family physician practices were assigned to begin the study in either the intervention or control phase.

Patients whose family physician was participating in the study were approached for recruitment upon presentation to the emergency department between I8 June 2001 and 2 April 2002. For their visit to be eligible, patients had to be 18 years of age or older, have been seen by the participating family physician at least once within the previous 2 years, and be able to provide informed consent as evaluated by the Short Portable Mental Status Questionnaire. ${ }^{11}$ Recruitment occurred on weekdays between 0800 and 2200 except on statutory holidays. Participants agreed to have their clinical information extracted from their emergency department medical chart and sent to their family physician.

Each of the 4 crossover periods was io weeks long. During each period, the results of the patient's visit were communicated to the participating family physician by way of either the intervention strategy or the control (usual practice) strategy depending on the physician's allocation at the time of the visit. At the completion of the study, all family physicians had received results by both strategies.

When family physicians were in the intervention phase, they received detailed clinical information of their patients' visit to the emergency department through a secure Webbased standardized communication system (SCS). The SCS program automatically issued advisory e-mails once per day (at 0700) to all family physicians whose patient or patients had presented to the emergency department within the previous 24 hours. The e-mail also provided a link to a secure Web page where the family physician could view and print a medical report with details of the emergency department visit, including the patient's name, presenting symptoms, emer- gency department diagnosis, disposition (room assignment if admitted to hospital), specialty consultation reports, laboratory test and electrocardiography results, imaging reports, discharge planning information and suggested follow-up, as well as any new medications or modifications to existing medication regimens. Emergency physicians were aware of which patients were having SCS reports issued because the names of the family physicians receiving SCS reports were posted in the emergency department.

When family physicians were in the control phase, they received a carbon copy of the first page of the emergency physician's notes by regular mail within I-2 weeks of the visit to the emergency department, the standard practice at the SMBD-JGH emergency department.

Resource utilization was defined in both settings as aspects of health care provision that consumed manpower and materials. The primary outcome or measure of resource utilization included repeat visits to the emergency department within I4 days of the initial visit; secondary outcomes in the emergency department included repeat visits within 28 days, admissions to hospital, mean length of stay in the emergency department, and requests for laboratory tests, imaging and specialty consultations. These data were collected through the hospital's database. Other secondary outcomes, which measured resource utilization in the family physician's office at the follow-up visit, included requests for laboratory tests, imaging and specialty consultations, and duplication of these requests with those made in the emergency department; these were obtained through a questionnaire sent by e-mail to the family physician 2I days after the patient's visit to the emergency department.

The post-visit questionnaire also asked physicians whether data regarding the patient's visit to the emergency department had been received, and received within 48 hours of that visit. Physicians were then asked to evaluate the legibility, comprehensiveness and usefulness of the data received as well as the knowledge gained regarding the patient's visit as a result of that data; these variables were evaluated using a 5-point Likert scale, with I representing the extreme negative (e.g., "strongly disagree" that the data were legible), 3 representing neutrality, and 5 representing the extreme positive (e.g., "strongly agree" that the data were legible).

On the basis of a rate of repeat visits within I4 days deemed to be the most important component of resource utilization and the one most open to influence - of $14 \%$ (obtained from the hospital database), a power of $80 \%$ and a significance level of $5 \%$, we calculated that 95I patient visits per study arm were necessary to detect a minimally important relative difference in repeat visits of $30 \%$ (i.e., $4.2 \%$ absolute difference). Adjustment for a cluster effect without the benefit of a precise estimate of intraclass correlation raised the estimated final sample to 2000 patient visits.

The crossover design was chosen as a means of enhancing the statistical power of the study. The study was budgeted to run over a Io-month period, and we chose crossover periods of to weeks each to ensure that each period was long enough for physicians to adapt to the communication strategy being used and yet short enough to allow 4 periods in total. 
All analyses were based on the intention-to-treat principle (i.e., irrespective of family physician compliance with the SCS intervention). Data were collected on each patient's visit, which created clusters associated with each family physician. Correlation of measurements within each family physician cluster was taken into account in all analyses. Repeatedmeasures techniques were also used to account for multiple emergency department visits by patients. The Generalized Estimating Equations approach (for binary outcomes, counts) ${ }^{12}$ and Mixed Model Analysis (for continuous outcomes) ${ }^{13}$ were used to account for the intracluster correlation and repeated measurements. The working correlation structure was set to be compound symmetry (or exchangeable form). Because of the 4-period crossover study design, 2 lag effects (first and second crossovers from the control to the intervention phase) and 2 washout effects (first and second crossovers from the intervention to the control phase) were also included in the model as potential confounders. The differences in outcomes between intervention and control phases were assessed through mean differences or odds ratios with their corresponding $95 \%$ confidence intervals. The odds ratios are presented as the odds of an outcome associated with increased resource utilization (i.e., increased number of repeat visits, tests and consultations) during the intervention phase over the odds of this same outcome during the control phase.

For the post-visit questionnaire sent to family physicians, variables scored using a 5-point Likert scale are reported as dichotomized variables whereby a score of 4 or 5 was counted in the affirmative and $\mathrm{I}-3$ in the negative.

The research protocol of this study was approved by the SMBD-JGH Research and Ethics Board.

\section{Results}

There were 43 family physicians whose clientele accounted for at least Ioo visits to the SMBD-JGH emergency department in the 12 months before the launch of the study and who were asked to participate (Fig. I). Twenty-three (53\%) agreed; most of those who refused cited a lack of interest and time. Of participating physicians, $86 \%$ were men, and their mean age was 54 (standard deviation [SD] 7) years; only $14 \%$ reported having "very much experience" with computers. According to self-report, $57 \%$ had a solo practice, the mean length of time in practice was 24 (SD 6) years, the mean proportion of their patients with a chronic medical condition was $49 \%$, and the mean proportion of their patients 70 years or older was 33\% (online Appendix I, available at www.cmaj.ca /cgi/content/full/174/3/313/DCI).

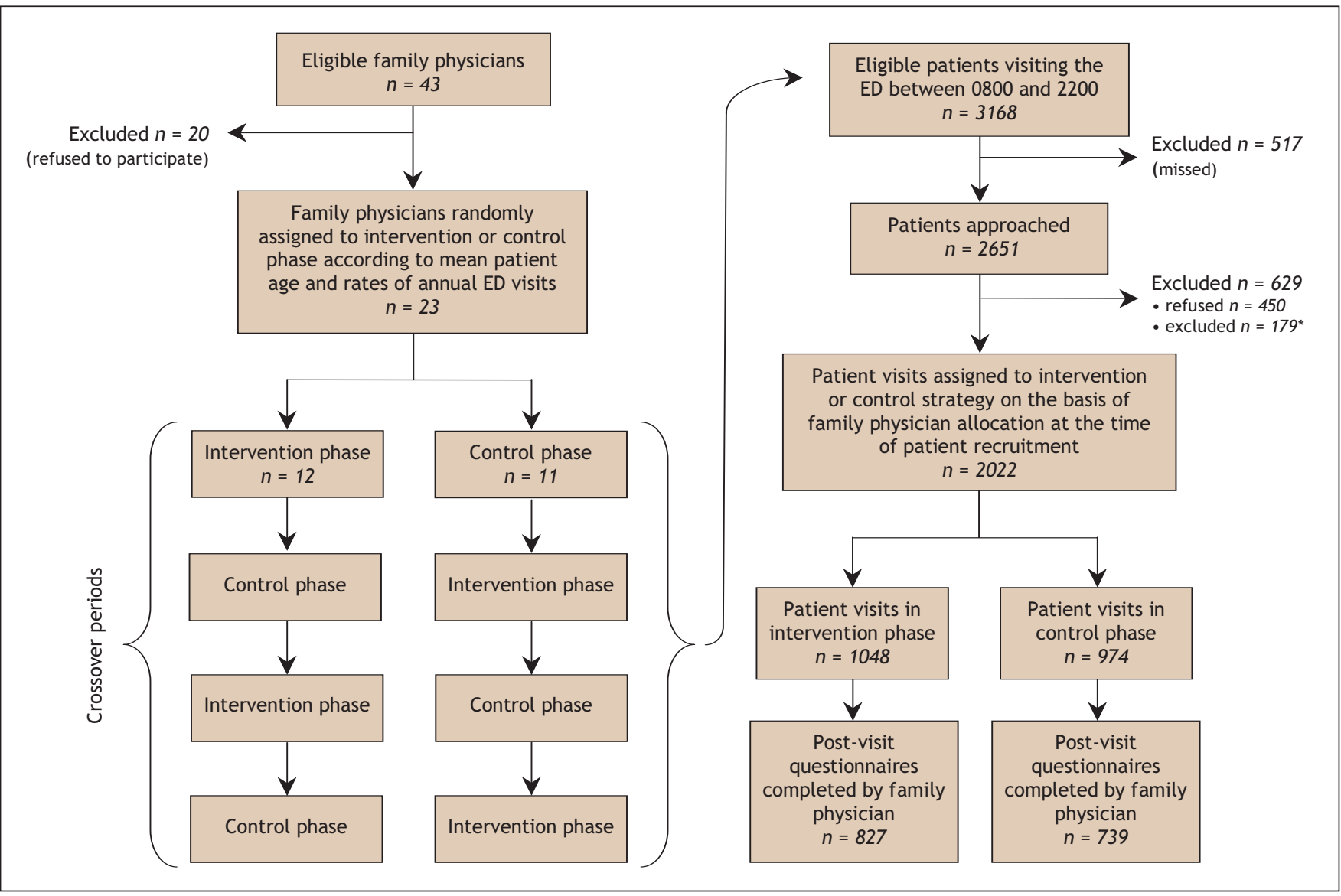

Fig. 1: Flow of participants through the study. The left-hand side of the flow chart depicts the flow of physicians, and the right-hand side depicts the flow of patients and patient visits during the 4 crossover periods. *A total of 179 patients were excluded because the patient was in an altered mental state $(n=129)$ or a state of agitation $(n=21)$ or because of a language barrier $(n=29)$. 
Table 1: Comparison of characteristics of patient visits to the emergency department (ED) in the intervention and control phases

\begin{tabular}{lcc}
\hline & \multicolumn{2}{c}{ Patient visit; no. $(\%)^{*}$} \\
\cline { 2 - 3 } Characteristic & $\begin{array}{c}\text { Intervention } \\
n=1048\end{array}$ & $\begin{array}{c}\text { Control } \\
n=974\end{array}$ \\
\hline Women & $611(58)$ & $551(57)$ \\
\hline Men & $437(42)$ & $427(43)$ \\
\hline Arrived in ED by ambulance & $221(21)$ & $194(20)$ \\
\hline Patient age, mean (SD), yr & $62.1(20.3)$ & $62.1(20.4)$ \\
\hline Triage level 1 or 2 & $276(26.3)$ & $280(28.7)$ \\
Chief symptom (most frequent) & $117(11.2)$ & $138(14.1)$ \\
\hline Chest pain & $100(9.5)$ & $97(9.9)$ \\
\hline Abdominal pain & $76(7.3)$ & $79(8.1)$ \\
\hline Dyspnea & &
\end{tabular}

Note: $\mathrm{SD}$ = standard deviation

*Unless stated otherwise.

A total of II family physicians were randomly assigned to the initial control group and $\mathrm{I} 2$ family physicians to the initial intervention group. During the ro months of recruitment, there were 3168 visits to the emergency department between 0800 and 2200 by patients whose family physician was participating in the study. In $265 \mathrm{I}(84 \%)$ of these visits, patients were asked to participate. The final sample is composed of $2022(76 \%)$ visits representing I $6 \mathrm{I} 6$ patients.

A comparison of the baseline characteristics of emergency department visits in the intervention and control arms suggests an even distribution of important variables (Table I). A total of 2022 questionnaires, one for each patient visit, were e-mailed to family physicians 2 I days after the visit. The overall response rate was $77 \%$ ( $79 \%$ in the intervention group and $76 \%$ in the control group). The individual response rate varied from $0 \%$ to $100 \% ; 3$ physicians were essentially unengaged in the trial and did not respond to any questionnaires. Excluding the 3 nonrespondents, the overall response rate increased to $88 \%$.

In evaluating the data received through the SCS and usual practice, more family physicians found the data received through the SCS legible, comprehensive and useful and as providing knowledge of the patient's emergency department visit (Table 2). However, the SCS failed to reduce the number of repeat visits to the emergency department within I4 days (97/8I4 patients in the intervention phase v. 88/802 in the control phase) and 28 days (I38/8I4 V. I35/802) as well as hospital admissions (259/1048 v. 224/974 patient visits) (Fig. 2). A post-hoc analysis was performed to determine whether the intervention had an effect on the number of repeat visits by patients who were more likely to leave the emergency department with a complex discharge plan (i.e., elderly patients). Although the difference did not quite achieve statistical significance, patients 65 years and over whose visit was communicated without the benefit of the SCS were noted to have a $30 \%$ relative increase in the risk of a repeat visit within
28 days of their initial visit. Other measures of resource utilization in the emergency department (online Appendix 2, available at www.cmaj.ca/cgi/content/full/174/3/313/DCI), including mean length of stay (I0.3 hours during the intervention phase v. I0.4 hours during the control phase) and number of tests ( 6.5 V. 7.0) and consultations (I.2 V. I.2) requested per visit did not result in significant differences.

Nor were significant differences seen in resource utilization in family physician offices at follow-up (online Appendix 3 , available at www.cmaj.ca/cgi/content/full/174/3/313/DCI) (e.g., 2 or more tests requested $[2.6 \%$ of patient visits during the intervention phase v. $1.7 \%$ of patient visits during the control phase], I specialty consultation requested $[4.3 \%$ v. $4.9 \%$ of patient visits]). There was no significant duplication of diagnostic tests between the emergency department and the family physician office during the intervention and control phases (24 v. 22, $p=0.93$ ), but there was significantly greater duplication in specialty consultations in the intervention phase than in the control phase (20 v. 8, $p=0.049$ ).

\section{Interpretation}

We hypothesized that the assured and expedient electronic transfer of clinical information from the emergency department to family physician offices for patients who had sought emergency care would lead to a reduction in resource consumption in the emergency department and at follow-up. From the emergency department perspective we anticipated that use of the SCS would promote timely follow-up of patients whose clinical summaries and discharge plans were communicated to their family physicians. This would, in turn, encourage less reliance on an emergency departmentbased work-up and lead to the discharge of patients from the emergency department who might otherwise have been admitted to the hospital because of uncertainty in postemergency department care. We also expected that the trans-

Table 2: Family physician evaluation of data regarding patients' emergency department (ED) visits received using the intervention and control strategies

\begin{tabular}{|c|c|c|}
\hline \multirow[b]{2}{*}{ Variable } & \multicolumn{2}{|c|}{ Patient visit; no. (\%) } \\
\hline & $\begin{array}{l}\text { Intervention } \\
n=827\end{array}$ & $\begin{array}{l}\text { Control } \\
n=739\end{array}$ \\
\hline Data on patient's visit to ED received & $768(93)$ & $461(62)$ \\
\hline $\begin{array}{l}\text { Data received within } 48 \mathrm{~h} \text { of patient's } \\
\text { ED visit }\end{array}$ & $546(66)$ & $4(0.5)$ \\
\hline Data is legible* & $652(78)$ & $207(28)$ \\
\hline Data is comprehensive* & $598(72)$ & $101(14)$ \\
\hline Data is useful* & $617(75)$ & $205(28)$ \\
\hline $\begin{array}{l}\text { Data provides knowledge of patient's } \\
\text { ED visit* }\end{array}$ & $650(79)$ & $202(27)$ \\
\hline
\end{tabular}

*These variables were evaluated using a 5-point Likert scale, where 1 represents the extreme negative (e.g., "strongly disagree" that the data received were legible), 3 represents neutrality and 5 represents the extreme positive. They are reported as dichotomized variables, whereby a score of 4 or 5 is counted in the affirmative and $1-3$ in the negative. 


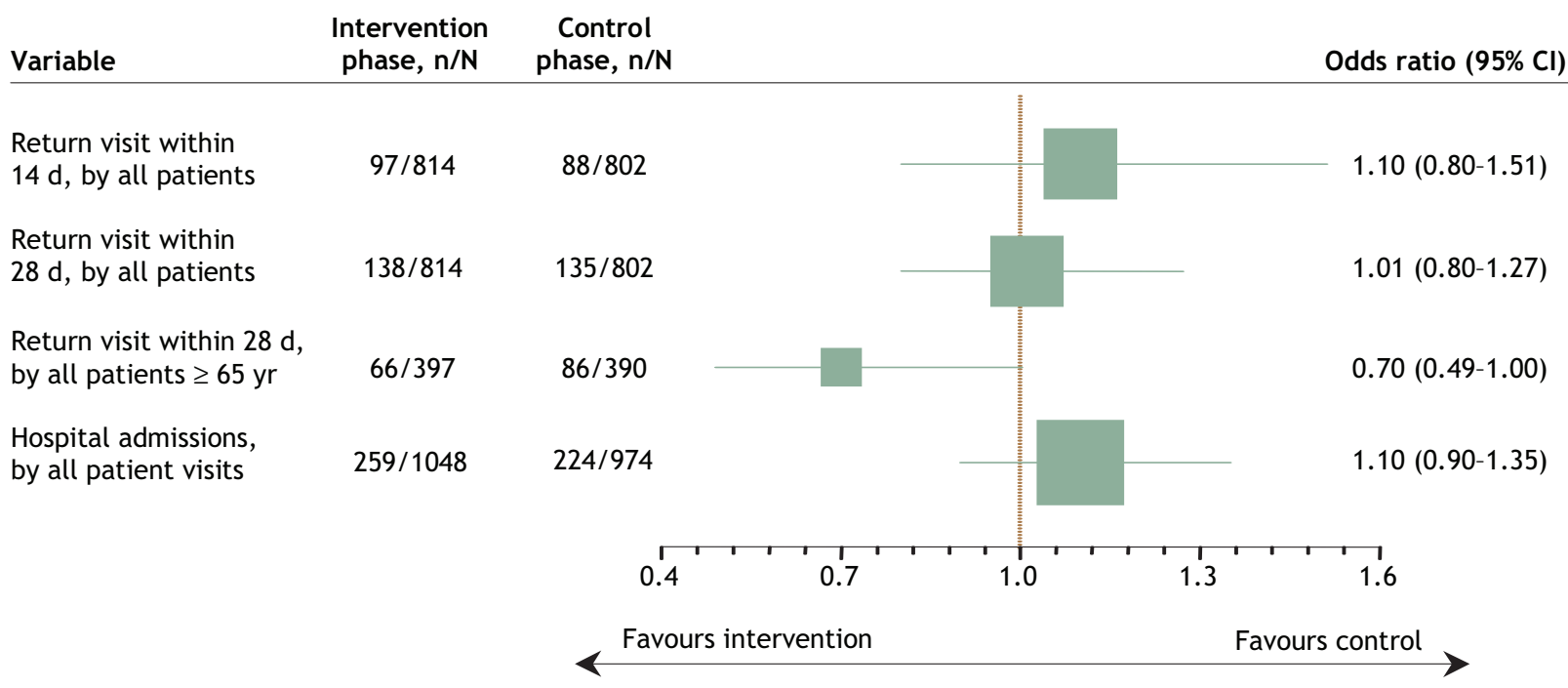

Fig. 2: Odds ratios of markers of resource utilization in the emergency department. $\mathrm{Cl}=$ confidence interval. The size of each box represents the sample size.

fer of all testing and consultation reports from the emergency department to family physicians would reduce duplication at follow-up. Most importantly, we expected the SCS to reduce the number of patients who were obliged to revisit the emergency department because of suboptimal post-visit management and possibly require admission. The SCS failed to yield benefits on all 3 fronts.

The lack of substantial benefit in all measures of resource consumption in the emergency department challenges our hypothesis that an SCS-like intervention will change emergency physician practices and limit in-hospital evaluations in favour of family physician follow-up for uncertain problems or minor illnesses. The lack of impact on duplication at follow-up suggests that family physician concerns, expressed in focus groups, about having to repeat testing and evaluations because they lack access to that information might have been overstated.

Another reason for a lack of effect might be the comparator used in this study. The SMBD-JGH already communicates patient information related to emergency department visits using carbon copies of the first page of the emergency department notes, and this standard practice was not suspended for the purpose of demonstrating the impact of the SCS intervention. This relatively strong comparator may have led to an underestimation of the impact of the SCS. A compliance analysis (not shown) based only on the physicians actively using SCS did not change the negative results regarding resource utilization.

A number of limitations of this study may have prevented an appreciation of the impact of the SCS intervention. For the intervention to be effective, emergency physicians had to take into account that patients for whom they were caring were going to have SCS reports issued to the family physician. Although we attempted to ensure this knowledge through education sessions and by posting the names of family physi- cians then in the intervention phase in prominent places throughout the emergency department, we do not know to what extent this knowledge was incorporated into emergency physician decision-making.

Measures of diagnostic test and specialty consultation requests and duplication in family physician offices at followup depended on accurate reporting by the family physicians when using the follow-up questionnaires. We encouraged family physicians to verify their files so as to be able to report actions taken with regard to being notified about an emergency department visit. However, the survey content was ultimately self-reported, and we have no definitive means of ascertaining what exactly was or was not done at follow-up.

The creation of the electronic health record and the move toward widespread electronic availability of patient data to all health care providers involved in a given patient's care is high on many national agendas. Both Canada ${ }^{14}$ and the United States ${ }^{15}$ have launched significant initiatives to create health information highways characterized by interoperability (i.e., the ability of multiple health care providers to access clinical information at the point of care). The financial justification for these systems has been made in terms of reducing administrative costs and the resources required for communicating the evaluative reports generated within the health care system. ${ }^{3}$ However, the assumptions underlying this cost benefit have been questioned. ${ }^{16}$

Fully standardized computerization of health care records has in general been noted to be effective in terms of the issuing reminders for preventive health tasks, increased prescribing of generic drugs and reduced duplication when computerized test ordering is performed. ${ }^{3}$ However, an electronic Web-based tool for transmitting clinical information from the emergency department to the primary care network does not appear to reduce resource utilization in either milieu. The cost of implementing electronic file-sharing between the 
emergency department and the primary care network does not appear to be recoverable through reduced length of stay, admission or repeat visit rates, or reduced duplication in test or consultation requests. Further studies are needed to assess the benefit of this intervention on indices of continuity and quality of care (medical error) and subsequent disease management by family physicians.

From the Emergency Department, Emergency Multidisciplinary Research Unit (Lang, Afilalo, Xue, Colacone, Léger, Rosenthal) and the Centre for Clinical Epidemiology and Community Studies (Vandal, Boivin, Shrier), Sir Mortimer B. Davis-Jewish General Hospital; and the Departments of Mathematics and Statistics (Vandal) and of Epidemiology and Biostatistics (Boivin), McGill University, Montréal, Qué.

Competing interests: None declared.

Contributors: Eddy Lang, Marc Afilalo, Alain C. Vandal, Jean-François Boivin, Antoinette Colacone and Ian Shrier were responsible for the conception and design of the study. Ruth Léger was responsible for data acquisition. Xiaoqing Xue, Eddy Lang and Alain C. Vandal analyzed and interpreted the data. Eddy Lang and Ruth Léger drafted the article. Xiaoqing Xue, Eddy Lang, Marc Afilalo, Alain C. Vandal, Jean-François Boivin, Antoinette Colacone and Ian Shrier critically revised the manuscript for important intellectual content. Xiaoqing Xue and Alain C. Vandal were responsible for statistical analysis. All of the authors approved the final version for publication.

Acknowledgements: We dedicate this research paper to the memory of Sophie Lapierre, PhD, from École Polytechnique, Université de Montréal. Dr. Lapierre's expertise in informatics was invaluable to the development of the SCS application.

We also thank Dr. Bernard Unger for his help in accessing the administrative databases, Dr. Howard Goldstein for facilitating the communication with family physicians and moderating the focus groups, Chantal Robitaille, $\mathrm{PhD}$, for her assistance in developing the questionnaires used in this study, and the medical, nursing and clerical staff of the SMBD-JGH Emergency Department for their support. Finally, we are also grateful for the continuous assistance provided by Mr. Frederic Abergel and his staff from the Informatics Systems Department of the SMBD-JGH.

This project was supported primarily by a grant from the Canadian Health Service Research Foundation (RCr 0249-45). The following organizations also contributed to study funding: Fonds de la Recherche en Santé du Québec, Ministère de la Santé et des Services Sociaux, Ministère de la Recherche de la Science et de la Technologie, Régie régionale de la Santé et des Services Sociaux, Association des Médecins d'Urgence du Québec, the SMBD-Jewish General Hospital Emergency Department and Information Services Department, the Brownstein Emergency Department Research Endowment Fund, the Armand Afilalo and Family Emergency Department Research Endowment Fund.

\section{REFERENCES}

I. Commission on the Future of Health Care in Canada. Building on values. The Future of health care in Canada - final report. 2002 Nov 28. Available: www.hc-sc .gc.ca/english/pdf/romanow/pdfs/HCC_Final_Report.pdf (accessed 2005 Dec I3).

2. Stiell AP, Forster AJ, Stiell IG, et al. Prevalence of information gaps in the emergency department and the effect on patient outcomes. CMAJ 2003;I69;I023-7.

3. Walker J, Pan E, Johnston D, et al. The value of health care information exchange and interoperability. Health Aff 2005 Jan I9. Epub (Web exclusive). DOI: I0.I377/ hlthaff.w5. Io

4. Teich J. The benefits of sharing clinical information. Ann Emerg Med I998;31:274-6.

5. Van Walraven C, Rokosh E. What is necessary for high-quality discharge summaries? Am J Med Qual 1999;I4:I60-9.

6. Wass AR, Illingworth RN. What information do general practitioners want about accident and emergency patients? J Accid Emerg Med i996;13:406-8.

7. Branger PJ, van der Wouden JC, Schudel BR, et al. Electronic communication between providers of primary and secondary care. BMJ I992;305:I068-70.

8. Stiell AP, Forster AJ, Stiell IG, et al. Maintaining continuity of care: a look at the quality of communication between Ontario emergency departments and community physicians. Can J Emerg Med 2005;7:155-6r.

9. Nelson S, Lang G. Virtual records. Using an Internet based system to manage patient records. Nephrol News Issues 2002;I6(I2):76-9.

Io. Kernan WN, Viscoli CM, Makuch RW, et al. Stratified randomization for clinical trials. JClin Epidemiol I999;52:19-26.

II. Pfeiffer E. A short portable mental status questionnaire for the assessment of organic brain deficit in elderly patients. J Am Geriatr Soc 1975;I0:433-4I.

I2. Liang KY, Zeger SL. Longitudinal data analysis using generalized linear models. Biometrika I986;73:13-22.

I3. Littell RC, Milliken GA, Stroup WW, et al. SAS system for mixed models. SAS institute Inc. Cary, NC; 2000.

I4. Giokas D. Canada health infoway - Towards a national interoperable electronic health record (EHR) solution. Stud Health Technol Inform 2005;II5:108-40.

I5. Huang EW, Hsiao SH, Liou DM. Design and implementation of a web-based HL7 message generation and validation system. Int J Med Inform 2003;70:49-58.

I6. Mitchell E. Sullivan F. A descriptive feast but an evaluative famine: systematic review of published articles on primary care computing during I980-97. BMJ 200I; 322:279-82

Correspondence to: Dr. Eddy Lang, Emergency Multidisciplinary Research Unit, Emergency Department, Jewish General Hospital, 3755 Côte St-Catherine, D-oro, Montréal QC H3T IE2; eddy.lang@mcgill.ca

\section{Editor's take}

- When a patient leaves the emergency department, 2 things have changed: the patient's state of health and the current information about that state. The information rarely accompanies the patient, and this dissociation is a loss to the patient and to the health care network; it can be dangerous as well.

- The authors of this study attempted to improve this disconnect by transmitting all of the information about the emergency room visit electronically to the patient's family doctor. Although the family doctors appreciated the enhanced information, the benefit that the authors anticipated, reduced resource use, did not accrue.

Implications for practice: Efforts to close the information gap make intuitive sense. More research needs to be done on what the key elements of that information are and how best to ensure their transmission to key people in the care network. 